\title{
Simulation of polyelectrolyte solutions. The density of bound ions
}

\author{
J.M.G. Sarraguça *, A.A.C.C. Pais \\ Departamento de Química, Universidade de Coimbra, Rua Larga, 3004-535 Coimbra, Portugal \\ Received 7 May 2004; in final form 9 September 2004 \\ Available online 5 October 2004
}

\begin{abstract}
We discuss a simple approach to describe the ion density around a polyelectrolyte chain, quantifying bound and bulk counterions, and allowing for the renormalization of the charge in the polyion. This approach is both physically motivated and readily extensible to systems containing other types of highly charged ions. The method addresses the problem in simulation experiments and allows to correlate ion condensation and compaction.

(C) 2004 Elsevier B.V. All rights reserved.
\end{abstract}

\section{Introduction}

Coulomb interaction and thus the counterion atmosphere and the induced degree of neutralization are to a large extent responsible for the behavior of highly charged polymers such as RNA and DNA. The difficulty of quantitatively assessing the ion density about the chain is both experimental [1,2] and theoretical $[3,4]$. The simplicity of the Debye-Hückel and Poisson-Boltzmann (PB) approximations, which may fail in some cases (especially in highly correlated systems with multivalent ions $[5,6])$, make them prevalent and, in biophysics, the Manning-Oosawa [7] counterion condensation (CC) and PB theories are still of major importance. For infinitely long rigid rods, Manning's two-state picture finds an exact counterpart in the exact solution of the cylindrical PB equation [8]. Extension of the PB equation for dilute solutions of finite length rod-like polyelectrolytes implies the use of a modified CC theory [9].

Studies on ion condensation are not only an attempt to provide a reference point [10] in polyelectrolyte solutions corresponding to the Debye-Hückel limiting law, but have a counterpart in the chain conformational behavior. The coil-globule coexistence phenomenon

\footnotetext{
${ }^{*}$ Corresponding author. Fax: +351 239827703.

E-mail address: jsarraguca@qui.uc.pt (J.M.G. Sarraguça).
}

and compaction behavior of DNA under various charged compaction agents are clearly linked to changes in electrostatic interactions (due to salt valence and concentration) and to variations in the ionic atmosphere in the close vicinity of the polyion.

The number of ions around the chain may be directly counted [11] in simulations, but the distinction between bound ions and those in the bulk is not clear, being the ion density established as a function of the distance from the chain. This distance concept has motivated several approaches, in terms of what is usually designated as Manning radius (see e.g. [12]). The Manning radius $\left(R_{\mathrm{M}}\right)$ is associated, for cylindrical symmetry around a linear polyion, with the axial distance that encloses the fraction of bound ions, but can be extended to other central colloidal particles [13]. Two major approaches have been used to establish $R_{\mathrm{M}}$ : (i) the search for a characteristic point [12,13], such as an inflection point in the running coordination number (see below) curve, $\mathrm{RCN}(r)$, or (ii) a physically motivated criterion for a priori assesment of $R_{\mathrm{M}}$ [14-17]. The first approach implies that the separation surface between bound and bulk ions should impart some alteration in the RCN behavior. The alteration would thus be visible in representations of $\mathrm{RCN}$ vs $1 / r$ [13] or $\ln r$ [12] based on the PB framework but considered to apply beyond this framework. In the a priori vision $R_{\mathrm{M}}$ may correspond to the value 
[15] that directly yields the Manning fraction of charge in the polyelectrolyte

$f=\frac{\varepsilon k_{\mathrm{B}} T R_{b b}}{Z q^{2}}$.

In Eq. (1) $R_{b b}$ is the average distance between chain beads, $Z$ is the charge of the cation, and all other symbols have the usual meaning. Also, $R_{\mathrm{M}}$ can be established directly in terms of Debye screening length or binding energy (see e.g. $[14,18])$. $R_{\mathrm{M}}$ as defined in the latter approaches does not usually correspond to special characteristics in the ordinary distributions.

In spite of some debate, the general aspects of the salt effect have been characterized, and are now consensual. Salt screens the charged interactions and contracts the polyelectrolyte chain up to a point. The discrepancy between $\mathrm{CC}$ theory and experiment (for instance in what concerns the osmotic behavior of DNA [19]) is thus sometimes attributed to the fact that for flexible chains counterion condensation alters the chain structure [11], but the neglect of molecular features, such as the finite radius is also mentioned $[8,20]$.

Some of the results of CC theory have been, in contrast, confirmed both from experiment and simulation. Ion condensation has been shown to be both salt and chain conformation independent [21], in certain regimes, and the onset of condensation [11] resides approximately where $\mathrm{CC}$ theory predicts. Also, it is clear that $\mathrm{CC}$ theory can be substantiated through statistical mechanics [22].

Most of this controversy arise due to the consideration of different concentration regimes and accuracy requirements. In the case of computer experiments, it is clear that there is no unequivocal way to establish the fraction of counterions, including salt ions, associated with the chain. The standard chain(bead)-ion distribution functions or the derived RCN (see below) show no clear cut-off between bound and bulk ions, especially for semi-flexible polyelectrolytes in which even a radial distance away from the backbone cannot be asserted. Also, the dimensions of the coil often overlap to a large extent to those where the most interesting features based on the chain-ion distance can be found.

In this Letter we suggest a new method to determine the bound fraction of ions based on the probability density function for the nearest-neighbor ion-ion distribution of the cations. This function reflects the effect of the presence of the polyion, which concentrates ions of opposite charge in its vicinity. The method corresponds to a density analysis of the ion cloud away from the polyelectrolyte backbone. It further characterizes the populations of bulk and bound ions, and gives some insight in the range of action of the chain over the neighboring ions. It also allows for a detailed analysis of such important factors as ion concentration and valence, and may prove very useful to pinpoint phenomena of charge inversion with physical significance. Finally, we have been able to establish partial fractions of condensed ions for two types of ions (mono and divalent, and mono and trivalent), for low concentrations of the multivalent ions.

\section{Model and simulation details}

The systems studied in this work were modeled within the primitive model and consist of charged hard-spheres residing in a continuum with a relative dielectric permitivity corresponding to that of water, $\varepsilon_{\mathrm{r}}=78.4$. The model is thoroughly described in reference [23].

The present study involves a relatively small semiflexible chain consisting of 100 negative beads of unitary charge, a bead radius $R_{\text {bead }}=2.0 \AA$, a bead-bead reference distance $r_{0}=5 \AA$, a bond force constant $k_{\text {bond }}=0.4$ $\mathrm{N} \mathrm{m}^{-2}$, an angular force constant corresponding to an intrinsic persistence length of $17 \AA$, and a reference angle $\alpha_{0}=180^{\circ}$. These values have previously been shown as adequate to describe a semi-flexible chain $[23,24]$. Counterions of unitary charge, salt cations of different valences and coions of unitary charge are characterized by the radii $R_{i}=2.0,2.5$ and $2.0 \AA$, respectively. The radius of the cations of monovalent salt was kept equal to that of the polyelectrolyte counterions, rendering them undistiguishable.

The concentration of particles in solution is determined by the radius of the simulation cell, $R_{\text {cell }}=340$ $\AA$. The relative concentration of salt and polyion is represented by the charge ratio, $\beta$,

$\beta=\frac{Z_{\text {cation }, h} \times N_{\text {cation }}}{N_{\text {bead }}}$,

where $Z_{\text {cation, } h}$ is the valence of the highest valence cation present, and $N_{\text {cation }}$ the number of these cations.

A number of different systems was simulated, varying the added salt (1:1, 2:1 and 3:1) and the charge ratio. For systems with di- and trivalent salt, $\beta$ ranges from 0.3 to 3 , whereas for monovalent ions the smallest value is $\beta=1$ and corresponds to the absence of salt, according to Eq. (2). The polylectrolyte counterions are thus included for the calculation of the charge ratio when only monovalent ions are present. All particles were considered explicitly.

The model was solved by Monte Carlo simulation in the canonical ensemble using the Molsim [25] package. The efficiency of the simulations was improved by taking concerted moves in the chain particles. These included slithering [26], pivot rotation and chain translation. Additionally, we employed a cluster-move [27] technique that is extremely important for achieving a better sampling efficiency, specially in systems where trivalent salt is present [23]. The number of MC steps 
used is about 50 times higher the statistical inefficiency parameter [28], which guarantees well converged results.

Analysis included standard particle-particle radial distribution functions, RCN and nearest-neighbor distance distribution functions. The former corresponds to the ratio between the average number density at a specific distance $r$ from a particle and the density at a distance $r$ from a particle in a random system with the same overall density; RCN gives the average number of particles, of a certain type, found within a specified distance from the reference particles; the nearest-neighbor distribution yields the probability density functions of distances between closest particles of chosen types.

\section{Results and discussion}

Standard bead-cation distribution representations (see Fig. 1a) or even RCN (Fig. 1b) provide single maximum or monotonic functions of the corresponding separation showing no clear-cut discrimination between bound and bulk ions (see discussion in Section 1), especially when dealing with semi-flexible polymers in which the direction away from the backbone is not well defined.
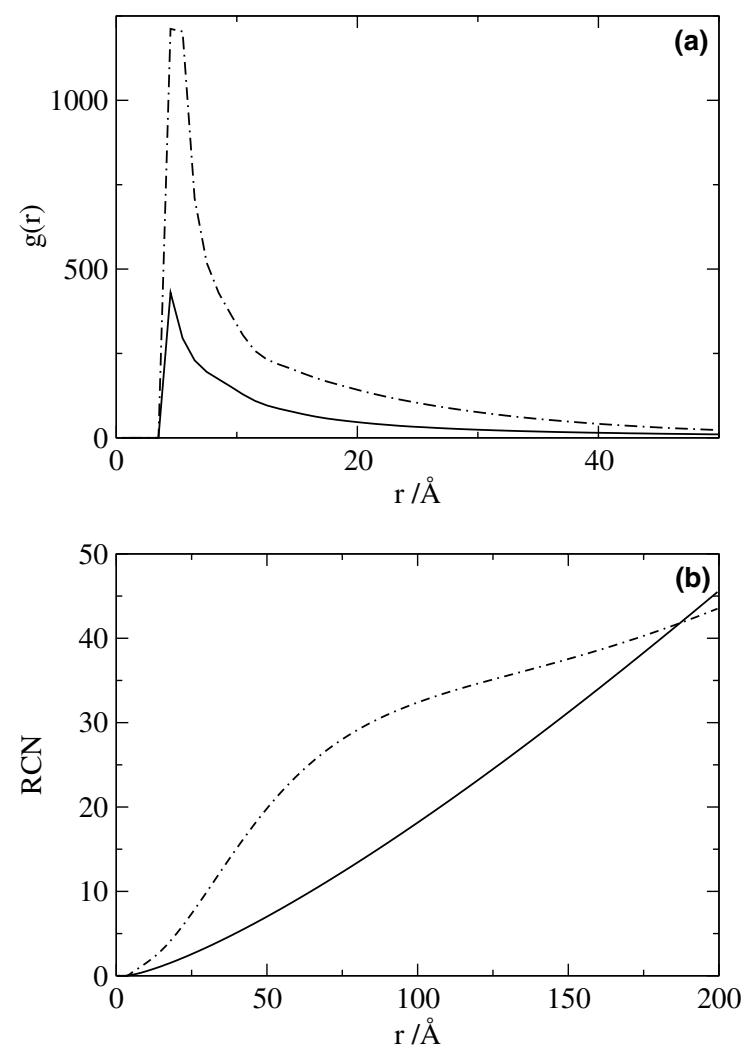

Fig. 1. Radial distribution function (a) and running coordination numbers (b) for charged bead-cations in the absence of added salt (full line) and charged bead-trivalent ions with $\beta=3$ (dash-point).
Some experimental properties (such as the rates of energy transfer from a electronically excited species to a ground-state acceptor) are strongly dependent on the nearest-neighbor average distances [29]. For homogeneous solutions, simple numerical calculations show that this distance, $\langle d\rangle$, is given by the following expression dependent on the number of particles $N_{\mathrm{p}}$ and the total volume $V_{\text {tot }}$ (or the related volume density)

$\langle d\rangle \approx\left(V_{\mathrm{tot}} / N_{\mathrm{p}}\right)^{1 / 3} / 1.79$.

In the presence of a negatively charged polyion, there is a crowding of the positive ions around the chain, but the bulk still follows closely Eq. (3). This originates a fluctuation in the ion density which can be detected in the cation-cation nearest-neighbor distribution (Fig. 2). In most cases, this distribution displays two maxima for $\beta \geqslant 1$, while for multivalent ions with smaller values of $\beta$ a single maximum reflects total or near-total condensation.

In Fig. 2 we can observe the cation-cation nearestneighbor distributions for monovalent $(\beta=1)$ and trivalent ions $(\beta=3)$. These panels illustrate two extreme situations in the set of systems under study. In the first, although a clear change in slope can be discerned, a
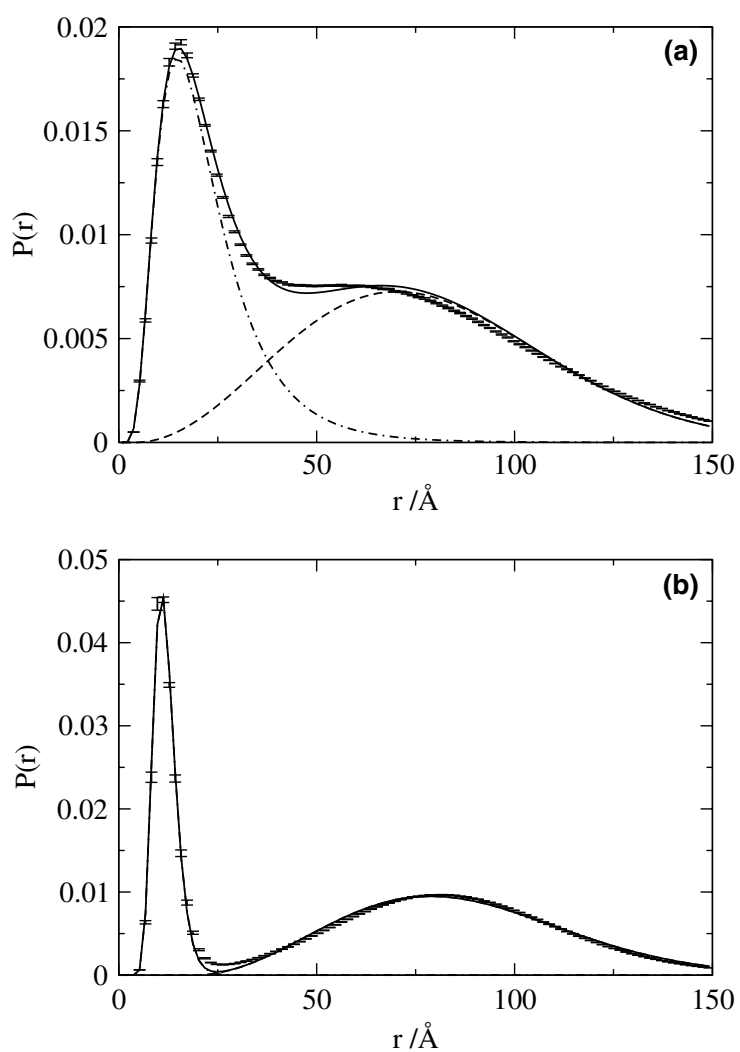

Fig. 2. Nearest-neighbor distributions for charged bead-cation in absence of added salt (a) and charged bead-trivalent ions with $\beta=3$ (b). The results obtained from simulation are presented by the error bars, the total fitted curve by the full line, the condensed counterions by the point-dash and free ions by the dashed line. 
definite separation between the bound and free ions is absent. In the trivalent case, the two maxima are well pronounced and the probability very close to zero in the transition between the two situations. We note that all systems with $\beta \geqslant 1$ were characterized by the presence of two maxima in this kind of distribution, except for the one illustrated in Fig. 2a.

The log-normal and Maxwell curves have proved to be, through extensive tests, the most adequate for describing the properties of bound and bulk ions, respectively, using a least-squares procedure. This conclusion is based both in the value of the sum-of-squares and frequency of alternation of sign in residuals. These two probability density functions have the right characteristics for a correct description of the nearest-neighbor distributions. They are asymmetrical and unimodal, and the random variable may be positive. The overall function is obtained by weighing with the fraction present in each population

$$
\begin{aligned}
& P(x)=f_{\text {cond }} \times \frac{1}{S x \sqrt{2 \pi}} \exp \left(-\frac{\ln x-M}{2 S^{2}}\right)+\left(1-f_{\text {cond }}\right) \\
& \times \sqrt{\frac{2}{\pi}} a^{3 / 2}(x-b)^{2} \exp \left(-\frac{a(x-b)^{2}}{2}\right) .
\end{aligned}
$$

The parameter $f_{\text {cond }}$ may be related to $f$ in Eq. (1) simply through

$f=1-f_{\text {cond }} \beta$

if only one type of cation is considered bound.

Restrictions were imposed, in some cases, for parameter $b$ keeping the value above the cation-cation contact distance. The values of parameters obtained for $\beta \geqslant 1$ are gathered in Table 1. For these charge ratios and multivalent salt, the number of bound monovalent counterions is negligible. For the bulk ions, irrespective of $Z_{\text {cation, } h}$, the average value and the standard deviation decrease as $\beta$ increases. A larger number of positive ions tends to stay in bulk, as the chain can no longer accomodate them, leading to a larger density and thus to a closer proximity between particles. For the condensed ions, and increasing $\beta$, the general tendency is for a decrease in the average and standard deviation. These changes become irrelevant for larger values of $\beta$.

Observation of the fitted curves in Fig. 2 and corresponding plots for other systems (not represented) show that the distances of closest proximity for condensed ions decrease as $Z_{\text {cation, } h}$ increases. The attraction to the polyanion is larger, the ion cloud around the chain denser and, as we will see below, the conformation of the chain is more compact. This is also consistent with the lower average values of the distribution.

The trend of the closest proximity between ions in bulk (corresponding to the value of $b$ ) is not as well defined. However, for $\beta=3$ in which the separation between condensed and bulk ions is drastic, this distance seems to increase with $Z_{\text {cation, } h}$, as the result of a larger pair repulsion.

The average values of the distributions of free ions are very close to the predictions of Eq. (3), but tend to be lower due to a remnant attractive power of the chain upon cations. In the case of trivalent ions, the difference is negligible as expected from the high values of $f_{\text {cond }} \beta$.

In Fig. 3 we compare the cation-cation nearest-neighbor distribution for the divalent salt $(\beta=2)$, curve (a), with that found in two situations in which the polyelectrolyte is absent. In the first, curve (b), the concentration of the divalent salt is the same as that in the polyelectrolyte solution, while curve (c) corresponds to a salt concentration determined from the fraction of divalent ions released into the bulk, if the polyelectrolytes were present. Note the similitude between the part of curve (a) ascribed to bulk ions, and curve (c), except for an

Table 1

\begin{tabular}{|c|c|c|c|c|c|c|c|c|c|c|}
\hline$Z_{\text {cation }, h}$ & $\beta$ & $f_{\text {cond }}$ & $\mathrm{S}$ & M & $\mu_{1}$ & $\sigma_{1}$ & $a$ & $b$ & $\mu_{2}$ & $\sigma_{2}$ \\
\hline \multirow[t]{3}{*}{1} & 1 & 0.42 & 0.53 & 2.98 & 23.0 & 12.9 & $4.5(-4)$ & $4.0^{\mathrm{a}}$ & 74.9 & $\overline{31.6}$ \\
\hline & 2 & 0.25 & 0.49 & 2.85 & 19.4 & 10.1 & $8.6(-4)$ & 5.6 & 54.4 & 23.0 \\
\hline & 3 & 0.23 & 0.49 & 2.87 & 19.9 & 10.5 & $1.4(-3)$ & 9.5 & 43.1 & 18.2 \\
\hline \multirow[t]{5}{*}{2} & 0.3 & 1.00 & 0.48 & 3.06 & 24.0 & 12.2 & & & & \\
\hline & 0.6 & 0.87 & 0.37 & 2.75 & 16.7 & 6.3 & $1.3(-4)$ & $5.0^{\mathrm{a}}$ & 140.6 & 59.3 \\
\hline & 1 & 0.66 & 0.34 & 2.65 & 15.1 & 5.3 & $1.9(-4)$ & $5.0^{\mathrm{a}}$ & 114.8 & 48.4 \\
\hline & 2 & 0.35 & 0.31 & 2.57 & 13.8 & 4.4 & $4.1(-4)$ & $5.0^{\mathrm{a}}$ & 79.3 & 33.5 \\
\hline & 3 & 0.26 & 0.32 & 2.58 & 13.9 & 4.5 & $7.4(-4)$ & 11.3 & 58.8 & 24.8 \\
\hline \multirow[t]{5}{*}{3} & 0.3 & 1.00 & 0.44 & 3.35 & 31.3 & 14.7 & & & & \\
\hline & 0.6 & 1.00 & 0.33 & 2.88 & 18.9 & 6.5 & & & & \\
\hline & 1 & 0.88 & 0.26 & 2.49 & 12.4 & 3.3 & $6.2(-5)$ & $5.0^{\mathrm{a}}$ & 201.9 & 85.2 \\
\hline & 2 & 0.46 & 0.25 & 2.45 & 11.9 & 3.0 & $3.1(-4)$ & 15.0 & 90.9 & 38.4 \\
\hline & 3 & 0.31 & 0.24 & 2.44 & 11.8 & 2.9 & $5.2(-4)$ & 16.2 & 70.1 & 29.6 \\
\hline
\end{tabular}

Parameters obtained for Eq. (4)

Distances in $\AA$; values in parenthesis represent powers of 10. Averages and standard deviations are denoted $\mu_{i}$ and $\sigma_{i}$, respectively, for bound $(i=1)$ and bulk $(i=2)$ populations.

${ }^{\mathrm{a}}$ Imposed values. See text. 


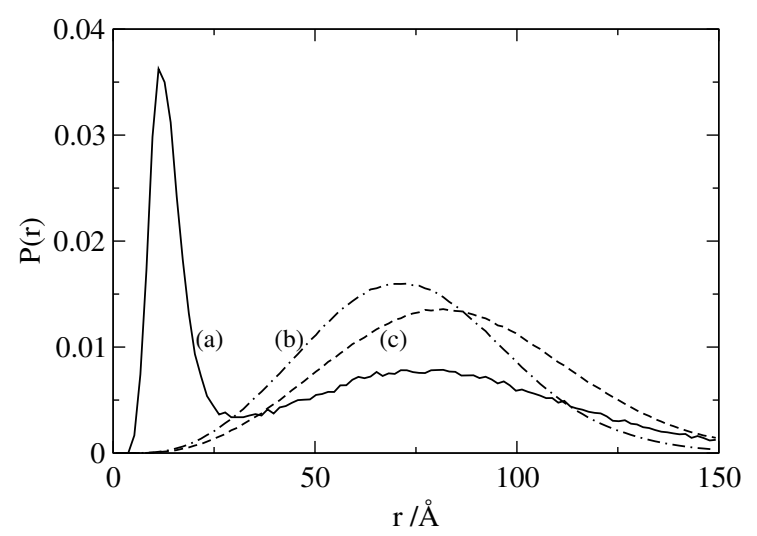

Fig. 3. Nearest-neighbor distributions of divalent cations in the presence (a) and absence (b) of a polyion, for a salt concentration corresponding to $\beta=2$. Line (c) represents the distribution in the absence of the polyion, with a salt concentration corresponding to the fraction of ions in bulk for system (a).

obvious normalization constant. The behavior of the distributions for different concentrations of salt in the absence of polyion (for small values of $r$ ) are in agreement with previous observation that ions of the same valence in different concentrations present an almost constant distance of maximum proximity.

Table 2 summarizes the estimates of the remaining fraction of charge in the chain using CC theory [7] and the method presented previously. $\mathrm{CC}$ theory provides reasonable estimates of the number of condensed ions, even for this finite semi-flexible chain, although some re-

Table 2

Estimate of the effective charge of the polyion expressed as the fraction $f$ of total charge, using $\mathrm{CC}$ theory and values from Table 1 .

\begin{tabular}{llll}
\hline$Z_{\text {cation }, h}$ & $\beta$ & $f$ & \\
\cline { 3 - 4 } & & CC & This work \\
\hline 1 & 1 & 0.67 & 0.58 \\
& 2 & 0.67 & 0.49 \\
& 3 & 0.67 & 0.32 \\
& 1 & 0.33 & 0.34 \\
& 2 & 0.33 & 0.29 \\
3 & 3 & 0.32 & 0.21 \\
& 1 & 0.21 & 0.13 \\
& 2 & 0.21 & 0.08 \\
& 3 & 0.21 & 0.06 \\
\hline
\end{tabular}

sults differ in almost 10 unitary charges. There is a consistent tendency for CC theory to overestimate the fraction of free ions, when compared both to the present and relevant experimental values. Other important aspect that emerges from this table is the trend of our simulation results, showing an increase in the condensed charge with increasing charge ratio, i.e., salt concentration.

In systems for which $\beta$ is clearly lower than unity, all multivalent and a fraction of the 'original' counterions of the polyion are condensed around the chain. This is also visible in the nearest-neighbor distributions for the latter. In these situations the chain backbone is partially neutralized by the multivalent charges and thus possesses a low residual linear charge density. The system for $\beta=0.6$ presents an extreme test to the method described. An estimate of the fraction of condensed counterions of the polyion was also obtained by the fitting procedure described earlier (results presented in Table 3). For the same charge ratio of di- and trivalent salt the distributions of bound and free counterions are very similar. This suggests that for $\beta<1$ the binding of monovalent counterions has the same characteristics, irrespective of $Z_{\text {cation, } h \text {. }}$

The polyion conformation is strongly dependent on the characteristics and concentration of the salt, as has been observed both from experiment, in a large number of articles, and theory (see e.g. [30,31]). The representation of the root-mean-square radius of gyration, $R_{\mathrm{g}}$, versus the remaining fraction of charge present in the chain (Fig. 4) shows that for multivalent salt there are two approximately linear regimes. For low values of $\beta$ and multivalent ions, the behavior is similar to that observed for monovalent cations, but when $\beta$ approaches unity there is an abrupt decrease on $R_{\mathrm{g}}$. For $\beta>1$ the slope of the curve tends to decrease again for all cations. In the case of trivalent ions, the decrease is less pronounced.

We also see that for the same $\beta$, and as the valence increases, the effective charge in the chain diminishes considerably. This is accompanied by a corresponding variation of $R_{\mathrm{g}}$. This is compatible with results previously obtained, in which for the same value of $\beta, R_{\mathrm{g}}$ has a tendency to decrease as $Z_{\text {cation, } h}$ increases (not to be confused with Fig. 1 in [30], in which an explicit charging of the monomers is performed).

Table 3

Parameters obtained for Eq. (4) corresponding to monovalent counterions $(\beta<1)$

\begin{tabular}{lllllllllll}
\hline$Z_{\text {cation }, h}$ & $\beta$ & $f_{\text {cond }}$ & $S$ & $M$ & $\mu_{1}$ & $\sigma_{1}$ & $a$ & $b$ & $\mu_{2}$ \\
\hline 2 & 0.3 & 0.31 & 0.62 & 3.36 & 35.0 & 24.2 & $5.1(-4)$ & 5.4 & 70.6 & 29.8 \\
& 0.6 & 0.12 & 0.62 & 3.53 & 41.2 & 28.1 & $5.6(-4)$ & $4^{\mathrm{a}}$ & 67.4 & 28.5 \\
3 & 0.3 & 0.30 & 0.63 & 3.36 & 35.3 & 24.7 & $5.0(-4)$ & 5.0 & 71.3 & 30.1 \\
& 0.6 & 0.16 & 0.64 & 3.74 & 52.0 & 37.2 & $6.2(-4)$ & 8.4 & 64.0 & 27.0 \\
\hline
\end{tabular}

\footnotetext{
Notation as in Table 1.
}

${ }^{a}$ Imposed value. See text. 


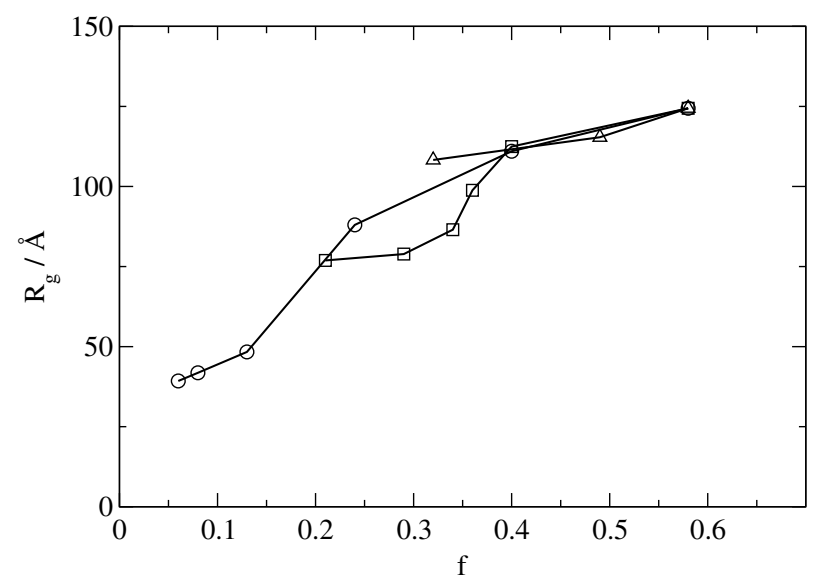

Fig. 4. Average radius of gyration as a function of the fraction of charge in the chain. Represented are different charge ratios for monovalent (triangles), divalent (squares) and trivalent (circles) salt.

In summary, irrespective of the valence, there is an increase in the number of condensed charges with concentration. The fraction of charge neutralized in the chain (and the degree of binding) are smaller for mono and divalent ions than that found for trivalent ions. Only the latter are capable of exceeding the $89-90 \%$ of neutralization, presented as the fraction necessary to induce compaction [32] in DNA.

\section{Conclusion}

The fraction of ions of any valence under the direct action of the polyelectrolyte backbone can be quantified and characterized in terms of average density and dispersion. The concentration of salt clearly influences this fraction in the concentration regimes under study, but results from the CC theory are acceptable estimates, even for this semi-flexible chain. Bulk ions behave very close to those in a solution in the absence of a polyelectrolyte, although its attractive influence can be discerned.

The conformational behavior of the chain depends strongly on the number of condensed ions, with a propensity for more compact structures, for each ion valence, as this number increases. The number of condensed ions as predicted by CC theory does not vary with salt concentration, making it impossible to correlate charge ratio $\beta$ and conformational indicators.

\section{Acknowledgment}

The authors thank Fundação para a Ciência e a Tecnologia (Portugal) for financial support through project POCTI/QUI/45331/2002.

\section{References}

[1] R. Das, T.T. Mills, L.W. Kwok, G.S. Maskel, L.S. Millett, S. Doniach, K.D. Finkelstein, D. Herschlag, L. Pollack, Phys. Rev. Lett. 90 (2003) 188103-1-4.

[2] T.G. Wensel, C.F. Meares, V. Vlachy, J.B. Matthew, Proc. Natl. Acad. Sci. USA 83 (1986) 3267.

[3] V.L. Murthy, G.D. Rose, Biochemistry 47 (2003) 14365.

[4] G. Ariel, D. Andelman, Europhys. Lett. 61 (2003) 67.

[5] L. Belloni, J. Phys. 12 (2000) R549.

[6] Y. Levin, Rep. Prog. Phys. 65 (2002) 1577.

[7] G.S. Manning, Acc. Chem. Res. 12 (1979) 443.

[8] M. Bret, B.H. Zimm, Biopolymers 28 (1984) 287.

[9] A. Deshovski, S. Obukhov, M. Rubinstein, Phys. Rev. Lett. 86 (2001) 2341.

[10] G.S. Manning, J. Chem. Phys. 51 (1969) 924.

[11] M.J. Stevens, S.J. Plimpton, Eur. Phys. J. B2 (1998) 341.

[12] M. Deserno, C. Holm., S. May, Macromolecules 33 (2000) 199.

[13] L. Belloni, Colloids Surf. A140 (1998) 227.

[14] M. Mandel, J. Phys. Chem. 96 (1992) 3934.

[15] M. Gueron, G. Weisbuch, Biopolymers 19 (1980) 353.

[16] G.R. Pack, G. Lamm, Int. J. Quantum Chem. 20 (1993) 213.

[17] G. Lamm, L. Wong, G.R. Pack, Biopolymers 34 (1994) 227.

[18] G.R. Pack, L. Wong, G. Lamm, Biopolymers 49 (1999) 575.

[19] P.L. Hansen, R. Podgornik, V.A. Parsegian, Phys. Rev. E 64 (2001) 021907-1-4.

[20] T. Alfrey Jr., P.W. Berg, H. Morawetz, J. Polym. Sci. 7 (1951) 543.

[21] P.A. Mills, A. Pashid, T.L. James, Biopolymers 32 (1992) 1491.

[22] U. Mohanty, B.W. Ninham, I. Oppenheim, Proc. Natl. Acad. Sci. USA 93 (1996) 4342.

[23] J.M.G. Sarraguça, M. Skepö, A.A.C.C. Pais, P. Linse, J. Chem. Phys. 119 (2003) 12621.

[24] A.A.C.C. Pais, M.G. Miguel, P. Linse, B. Lindman, J. Chem. Phys. 117 (2002) 1385

[25] P. Linse, Molsim, version 3.3.2, Lund University, Sweden, 2002.

[26] C. Brender, M. Lax, J. Chem. Phys. 79 (1983) 2423.

[27] H.L. Gordon, J.P. Valleau, Mol. Simulat. 14 (1995) 361.

[28] M.P. Allen, D.J. Tildesley, Computer Simulation of Liquids, Clarendon Press, Oxford, 1987.

[29] M.J. Tapia, H.D. Burrows, M.E.D.G. Azenha, M.G. Miguel, A.A.C.C. Pais, J.M.G. Sarraguça, J. Phys. Chem. B 106 (2002) 6966.

[30] M.O. Khan, B. Jönsson, Biopolymers 49 (1999) 121.

[31] F.J. Solis, O. de la Cruz, J. Chem. Phys. 112 (2000) 2030.

[32] S. He, P.G. Arscott, V.A. Bloomfield, Biopolymers 53 (2000) 329. 